\title{
THE STRUCTURE OF HYBRIDIN
}

B. Makhsudova, Z. P. Pakudina, and A. S. Sadykov

Khimiya Prirodnykh Soedinenii, Vol. 5, No. 4, pp. 322-323, 1969

By threefold chromatography in the butan 1-ol-pyridine-water $(6: 4: 3)$ and ethyl acetate-pyridine-water (2:1: 2) systems it has been established that the sugar obtained in the hydrolysis of hybridin with $3 \%$ hydrochloric acid contains galactose, glucose, and xylose.

In a quantitative determination of the aglycone of hybridin by the gravimetric method [1] and of the sugars by the aniline phthalate method [2] the ratio of quercetin to sugars was found to be $1: 3$ and that of xylose to galactose and glucose $1: 2$. Thus, the hybridin isolated from the flowers of hybrid hibiscuses [3] has the structure of not a bioside but a trioside of quercetin.

On the basis of the bathochromic shifts in the UV spectra obtained in the presence of ionizing and complex-forming reagents (sodium acetate, boric acid, aluminum chloride, sodium ethoxide), it has been shown that the sugar is attached to the quercetin in position 3. Quercetin 3-xyloside has been synthesized in the laboratory of polyene compounds of VNIVI [All-Union Scientific-Research Vitamin Institute] (Moscow) in collaboration with M. K. Shakova by the method of Ise and Wender [4]. On paper chromatography in the ethyl acetate-acetic acid-water $(3: 1: 3)$ system it had $R_{f} 0.86$, differing from the xyloside obtained by the stepwise hydrolysis of hybridin with $10 \%$ acetic acid.

It is most likely that hybridin is quercetin $3-[1 \rightarrow 3-(\beta-D-x y l o p y r a n o s i d o-\beta-D-$ gly ucopyranoside- $\beta-D-$ galactofuranoside]. Hybridin has also been isolated from the leaves of the cotton plant of type 108-F and from a hybrid of the cotton plant with hibiscus.

\section{REFERENCES}

1. B. Makhsudova, Z. P. Pakudina, and A. S. Sadykov, Tr. TashGU, 3, no. 341, 77, 1968.

2. G. I. Zaitseva and T. P. Afanas'ev, Biokhim., 22, 1035, 1957.

3. B. Makhsudova, Z. P. Pakudina, and A. S. Sadykov, KhPS [Chemistry of Natural Compounds], 3, $11,1967$.

4. C. H. Ise and S. H. Wender, J. Am. Chem. Soc., 74, 4606, 1952.

14 February 1969

Scientific-Research Institute for the Chemistry and Technology of Cotton Cellulose, Tashkent

UDC 547.972

\section{THE FLAVONOIDS OF ARTEMISIA DRACUNCULUS}

T. K. Chumbalov, M. M. Mukhamed"yarova, and O. V. Fadeeva

Khimiya Prirodnykh Soedinenii, Vol. 5, No. 4, p. 323, 1969

This paper gives the results of a study of the flavonoids from the epigeal part of Artemisia dracunculus L. (tarragon) growing in the southern regions of the USSR. The content of flavonoids in tarragon varies from 1.8 to $3.7 \%$ [1].

By adsorption chromatography on Kapron and preparative chromatography on paper using several systems of solvents, we have isolated three individual substances. From the results of chemical and spectral investigations, the first substance, with $\mathrm{mp} 304-305^{\circ} \mathrm{C}$, is quercetin and the second, with $\mathrm{mp} 238-240^{\circ} \mathrm{C}$ is hyperoside. The third substance with $\mathrm{mp} 155-$ $157^{\circ} \mathrm{C},[\mathrm{c}]_{\mathrm{D}}^{20}+82.6^{\circ}, \lambda_{\max } 358,253 \mathrm{~m} \mu$, has been characterized provisionally as isorhamnetin $7-\alpha-\mathrm{D}-\mathrm{galactopyran}-$ oside p-hydroxybenzoate. Alkaline saponification under mild conditions leads to the splitting off of p-hydroxybenzoic acid. Alkaline cleavage of the aglycone yielded vanillic acid and phloroglucionol. UV spectroscopy showed that in the glycoside the $5,3,4$-hydroxy groups are free.

REFERENCE

1. G. P. Pavelovskaya, L. S. Alyukina, and L. K. Khyshev, Vestn. AN KazSSR, no. 9, 269, 60, 1967. 